EPJ Web of Conferences 60, 13001 (2013)

DOI: $10.1051 /$ epjconf $/ 20136013001$

(C) Owned by the authors, published by EDP Sciences, 2013

\title{
Heavy-flavour measurements in Pb-Pb collisions with ALICE at the LHC
}

\author{
Chiara Bianchin 1, a \\ ${ }^{1}$ Utrecht University, Princetonplein 5, 3584 CC Utrecht, The Netherlands
}

\begin{abstract}
The ALICE experiment studies the properties of the strongly-interacting matter created in high energy heavy-ion collisions, called Quark-Gluon Plasma (QGP). Heavy quarks are a powerful probe for investigating such a state of matter, since they are predominantly produced in the first hard scattering processes and they bring to the final state information on the deconfined phase. Heavy-flavour particles are reconstructed via hadronic and semi-leptonic decays in the ALICE detector. The measurements of the modification of the heavyflavour hadrons transverse momentum distribution in $\mathrm{Pb}-\mathrm{Pb}$ collisions with respect to $\mathrm{pp}$ and of their azimuthal anisotropy show that heavy quarks have a sizeable interaction with the medium constituents.
\end{abstract}

\section{Introduction}

The ALICE experiment studies the properties of the strongly-interacting matter created in high energy heavyion collisions. This state of deconfined matter, called Quark-Gluon Plasma (QGP), is predicted by Quantum Chromodynamics (QCD).

Heavy quarks are a powerful probe for investigating such a state of matter, since they are predominantly produced on a short time scale in hard scattering processes during the early stage of the collision and are expected to be sensitive to its energy density, through the mechanism of parton energy loss. This QCD energy loss is expected to occur via both inelastic (medium-induced gluon radiation, or radiative energy loss) [1] and elastic (collisional energy loss) [2] processes. Quarks have a smaller colour coupling factor with respect to gluons and the 'dead-cone effect' should reduce small-angle gluon radiation for heavy quarks with moderate energy-over-mass values [3]. Hence, the energy loss for heavy quarks is expected to be smaller than for light quarks and the latter smaller than for gluons.

The energy loss and the transport coefficient (dynamical properties) of the QGP can be studied experimentally via the nuclear modification factor, $R_{\mathrm{AA}}$, which is the ratio of the yield measured in $\mathrm{Pb}-\mathrm{Pb}$ to that observed in $\mathrm{pp}$ scaled with the number of binary nucleon-nucleon collisions. The expected hierarchy in the energy loss translates into a hierarchy of $R_{\mathrm{AA}}$, namely $R_{\mathrm{AA}}(\mathrm{B})>\mathrm{R}_{\mathrm{AA}}(\mathrm{D})>$ $\mathrm{R}_{\mathrm{AA}}$ (light).

In nuclear collisions, anisotropic patterns of particle production originate from the initial anisotropy in the spatial distribution of the nucleons participating in the collision. The anisotropy is described by the coefficients $v_{n}=$ $\left\langle\cos \left[n\left(\phi-\Psi_{n}\right)\right]\right\rangle$ of the Fourier expansion describing the azimuthal angle $\phi$ of particles with respect to the initial state symmetry plane for the considered harmonic $\Psi_{n}$.

a. e-mail: chiara.bianchin@cern.ch
For non-central collisions the overlap region of the colliding nuclei has a lenticular shape and the anisotropy is dominated by the second coefficient $v_{2}$ or elliptic flow. At low transverse momentum $p_{\mathrm{T}}$, charmed hadron $v_{2}$ offers a unique opportunity to test whether quarks with large mass $\left(m_{c} \approx 1.5 \mathrm{GeV} / \mathrm{c}^{2}\right)$ participate in the collective expansion dynamics and possibly thermalize in the medium $[4,5]$. At low and intermediate $p_{\mathrm{T}}$, the heavy flavour particle elliptic flow is expected to be sensitive to the heavy-quark hadronization mechanism (recombination). At high $p_{\mathrm{T}}$, it can constrain the path-length dependence of parton energy loss, complementing the measurement of the nuclear modification factor $R_{\mathrm{AA}}$.

\section{Heavy-flavour measurements with the ALICE detector}

The ALICE detector is composed of a central rapidity region equipped with several detectors for vertexing, tracking and particle identification and a forward muon spectrometer [6]. In the central rapidity region, heavyflavour particles are measured via full reconstruction of $\mathrm{D}$ meson hadronic decay channels and via semi-electronic decay channels, while at forward rapidity they are accessible via the semi-muonic decay channel.

\subsection{D mesons}

The $\mathrm{D}^{0}, \mathrm{D}^{+}, \mathrm{D}^{*+}$, and $\mathrm{D}_{\mathrm{s}}^{+}$mesons and their antiparticles are reconstructed in the speudorapidity interval $|\eta|<$ 0.8 via their hadronic decay channels $\mathrm{D}^{0} \rightarrow \mathrm{K}^{-} \pi^{+}$(with branching ratio, $\mathrm{BR}$, of $3.88 \pm 0.05 \%), \mathrm{D}^{+} \rightarrow \mathrm{K}^{-} \pi^{+} \pi^{+}$ (BR of $9.13 \pm 0.19 \%), \mathrm{D}^{*+} \rightarrow \mathrm{D}^{0} \pi^{+}(\mathrm{BR}$ of $67.7 \pm 0.5 \%)$ with $\mathrm{D}^{0} \rightarrow \mathrm{K}^{-} \pi^{+}$, and $\mathrm{D}_{\mathrm{s}}^{+} \rightarrow \phi \pi^{+} \rightarrow \mathrm{K}^{-} \mathrm{K}^{+} \pi^{+}(\mathrm{BR}$ of $2.28 \pm 0.12 \%$ ) [7]. The $\mathrm{D}^{0}, \mathrm{D}^{+}$, and $\mathrm{D}_{\mathrm{s}}^{+}$mesons decay weakly with mean proper decay lengths $(c \tau)$ of approximately 123,312 , and $150 \mu \mathrm{m}$, respectively [7]. The 
$\mathrm{D}^{*+}$ meson decays strongly at the primary vertex. The D meson yields were measured with an invariant mass analysis of fully reconstructed decay vertices, selected by means of topological cuts and particle identification (PID). The impact parameter resolution needed to select tracks displaced by few hundred microns from the primary vertex is provided by the Inner Tracking System and in particular by the first two layers of Silicon Pixel Detectors. The azimuthal anisotropy measurement is performed with the event plane method. The D meson yield is extracted in two complementary $\pi / 2$-wide regions, in-plane (]$\left.\left.\left.\left.-\frac{\pi}{4}, \frac{\pi}{4}\right] \cup\right] \frac{3 \pi}{4}, \frac{5 \pi}{4}\right]\right)$ and out-of-plane (]$\left.\left.\left.\left.-\frac{\pi}{4}, \frac{3 \pi}{4}\right] \cup\right] \frac{5 \pi}{4}, \frac{7 \pi}{4}\right]\right)$, respectively with respect to the event plane $\psi_{2}$. $v_{2}$ can finally be calculated as

$$
v_{2}=\frac{1}{R_{2}} \frac{\pi}{4} \frac{N_{\text {in-plane }}-N_{\text {out-of-plane }}}{N_{\text {in-plane }}+N_{\text {out-of-plane }}}
$$

obtained by integrating the Fourier expansion. The factor $R_{2}$ is the correction for the finite resolution in the estimation of the symmetry plane $\Psi_{2}$ via the event plane $\psi_{2}$.

The measured $\mathrm{D}$ meson yield has a contribution from feed-down from B meson decays, which varies with the applied selections and amounts to about $10-20 \%$ in our analyses, depending on $p_{\mathrm{T}}$. This contribution is estimated with FONLL-based methods and cross-checked with a data-driven method exploiting the different impact parameter distribution of prompt and secondary D mesons [8]. The central values of both measurements assume an hypothesis on $R_{\mathrm{AA}}(\mathrm{D} \leftarrow \mathrm{B})$ and on $v_{2}^{\mathrm{D} \leftarrow \mathrm{B}}$, respectively. A systematic uncertainty was estimated by varying these hypotheses and the parameters used in the determination of the fraction of prompt D mesons with FONLL.

\subsection{Heavy-flavour decay electrons}

Two complementary electron identification approaches are used. Both approaches require that the specific energy loss, $\mathrm{d} E / \mathrm{d} x$, measured in the Time Projection Chamber (TPC), is compatible with the expected electron $\mathrm{d} E / \mathrm{d} x$. To increase the purity of the electron candidate sample, in the first approach the information from the Time-Of-Flight (TOF) detector is used, while, in the second approach, electromagnetic calorimetry (EMCal) is used. The $p_{\mathrm{T}}$ regions covered by the two analyses are $1.5<p_{\mathrm{T}}<6 \mathrm{GeV} / \mathrm{c}$ for the TPC-TOF approach and $2<p_{\mathrm{T}}<13 \mathrm{GeV} / \mathrm{c}$ for the TPC-EMCal.

In order to measure the $R_{\mathrm{AA}}$ and the $v_{2}$ of heavyflavour decay electrons (HFE) the background from several sources must be subtracted. A Monte Carlo (MC) based method with data-driven inputs, called "cocktail method", is used to determine the non HFE contributions. A large fraction of non-HFE electrons, the so-called photonic electrons, originates from photon conversion and Dalitz decays of $\pi^{0}$ and $\eta$. This contribution is also estimated with a data-driven method and subtracted. In this approach to estimate the "photonic" contributions, electrons are paired with an oppositely charged partner and a selection on the invariant mass of the $e^{+} e^{-}$pair below $0.05 \mathrm{GeV} / \mathrm{c}^{2}$ is applied. The low mass region is dominated by photonic electrons, while the remaining contribution from HFE is determined using the like-sign pair invariant mass distribution.

In the $R_{\mathrm{AA}}$ measurement the photonic and nonphotonic contributions are subtracted from the corrected inclusive spectrum to obtain the heavy-flavour electron spectrum. In the $v_{2}$ analyses, the $v_{2}$ of each background source is estimated with the cocktail using the ALICE data as an input whenever possible.

\subsection{Heavy-flavour decay muons}

Muon tracks are reconstructed in the 5 tracking chambers of the muon spectrometer. A hadron absorber is placed upstream from the spectrometer and a muon filter is situated between the tracking chambers and the two trigger stations. A selection on the distance of closest approach between the track and the primary vertex removes beamgas interaction background. The reconstructed tracks are matched with tracklets measured in the trigger stations to reject punch through hadrons. The main source of background left is due to muons from $\mathrm{K}$ and $\pi$ decays. $\mathrm{K}$ and $\pi$ are measured at mid-rapidity, extrapolated to $2.5<\eta<4$ as explained in [10], and then let decay (those hadrons decaying within the absorber are propagated using Geant). The background contribution is subtracted from the inclusive muon spectrum limiting the measurement to the region $p_{\mathrm{T}}>4 \mathrm{GeV} / \mathrm{c}$, where the contribution to the $\mu$ spectrum from heavy flavour (HFM) is dominant.

\section{Results and conclusions}

Figure 1 and 2 left panels show the average $\mathrm{D}$ meson $R_{\mathrm{AA}}$ in $0-7.5 \%$ central $\mathrm{Pb}-\mathrm{Pb}$ collisions and HFE $R_{\mathrm{AA}}$ in 0 $10 \%$, respectively, as a function of $p_{\mathrm{T}}$. A softening of the $p_{\mathrm{T}}$ distribution with respect to $\mathrm{pp}$ collisions is observed in both measurements. For D mesons, the suppression at high $p_{\mathrm{T}}$ reaches up to about a factor 5. Next-to-leadingorder pQCD calculations (MNR) [11] with EPS09 shadowing [12] cannot explain the suppression (cf. [8]). Other calculations are compared to the data and those that implement radiative energy loss supplemented with in-medium dissociation [13] and radiative plus collisional energy loss in the WHDG [14] and BDMPS-ASW [15] implementations describe the data reasonably well. The modification of the heavy-flavour $p_{\mathrm{T}}$ distribution in $\mathrm{Pb}-\mathrm{Pb}$ with respect to pp collisions looks similar to that of charged hadrons, according to the comparison of the two $R_{\mathrm{AA}}$ shown in [8] for $\mathrm{D}$ mesons. The suppression of heavy-flavour yield at high $p_{\mathrm{T}}$ increases going from peripheral to central collisions, as reported in the left panel of Fig. 3 for HFM and in the left panel of Fig. 4 for D mesons.

The heavy-flavour $R_{\mathrm{AA}}$ is similar in different rapidity regions as shown in the right panel of Fig. 3 where the measurement of $R_{\mathrm{AA}}$ vs. $p_{\mathrm{T}}$ of muons from heavy-flavour decays $R_{\mathrm{AA}}$ vs $p_{\mathrm{T}}$, performed in the forward rapidity region $2.5<y<4$, is compared to HFE $R_{\mathrm{AA}}$ at central rapidity.

A hint of mass ordering in the energy loss can be seen in the left panel of Fig. 4 where we compare the $R_{\mathrm{AA}}$, as 
a function of centrality, of $J / \psi$ from B, measured by CMS [9], to the $R_{\mathrm{AA}}$ of D mesons, which is systematically lower (the kinematic region of the two measurement is not exactly the same, though). The measurement of $\mathrm{D}_{\mathrm{s}}^{+} R_{\mathrm{AA}}$ is particularly interesting due to its constituent strange quark. If the hadronization of $\mathrm{D}$ mesons occur via recombination in the low-intermediate $p_{\mathrm{T}}$ region, a enhanced production of $\mathrm{D}_{\mathrm{s}}^{+}$is expected in $\mathrm{Pb}-\mathrm{Pb}$ collisions due to the abundance of strange quarks in the medium. In the right panel of Fig. 4 the $R_{\mathrm{AA}}$ of different $\mathrm{D}$ meson species are shown separately : $\mathrm{D}^{0}, \mathrm{D}^{+}$, and $\mathrm{D}^{*+}$ mesons lie on a similar trend, while the $\mathrm{D}_{\mathrm{s}}^{+}$seems to feature a larger $R_{\mathrm{AA}}$ at low $p_{\mathrm{T}}$, although the uncertainties are too large at present to allow us to draw any conclusions.

The D meson and HFE $v_{2}$ measurements are shown in Fig. 1 and 2, right panels. The average of the measured $\mathrm{D}$ meson $v_{2}$ values in the interval $2<p_{\mathrm{T}}<6 \mathrm{GeV} / \mathrm{c}$ is larger than 0 with $5.7 \sigma$ significance, suggesting that low momentum charm quarks take part in the collective motion of the system. A positive $v_{2}$ is also observed for D mesons with $p_{\mathrm{T}}>6 \mathrm{GeV} / \mathrm{c}$, which most likely is due to the pathlength dependence of the partonic energy loss, although the large uncertainties do not allow a firm conclusion to be made. The magnitude of the $\mathrm{D}$ meson $v_{2}$ is similar to that of light hadrons [16].

According to the results just shown, charm quarks interact with the medium similarly to light quarks. The comparison the measured $R_{\mathrm{AA}}$ and $v_{2}$ values to the theoretical predictions (Fig. 1 and 2) indicates that it is challenging for models to simultaneously describe the large suppression of heavy flavour yield in central collisions and their anisotropy in non-central collisions.

\section{Acknowledgements}

The European Research Council has provided financial support under the European Community's Seventh Framework Programme (FP7/2007-2013) / ERC grant agreement no 210223 . This work was also supported by a Vidi grant from the Netherlands Organisation for Scientific Research (project number 680-47-232).

\section{References}

[1] M. Gyulassy and M. Plumer, Phys. Lett. B 243 (1990) 432

[2] M.H. Thoma and M. Gyulassy, Nucl. Phys. B 351 (1991) 491

[3] Y.L. Dokshitzer and D. Kharzeev, Phys. Lett. B 519 (2001) 199

[4] S. Batsouli, S. Kelly, M. Gyulassy and J. L. Nagle, Phys.Lett. B 557 (2003) 26 [nucl-th/0212068].

[5] V. Greco, C. M. Ko and R. Rapp, Phys. Lett. B 595 (2004) 202 [nucl-th/0312100].

[6] K. Aamodt et al. [ALICE Collaboration], JINST 3 (2008) S08002.

[7] J. Beringer et al. (Particle Data Group), Phys. Rev. D86, 010001 (2012).

[8] B. Abelev et al. [ALICE Collaboration], JHEP 09 (2012) 112.

[9] CMS-PAS-HIN-12-014.

[10] B. Abelev et al. [ALICE Collaboration], Phys. Rev. Lett. 109 (2012) 112301.

[11] M. Mangano, P. Nason and G. Ridolfi, Nucl. Phys. B373 (1992) 295.

[12] K. J. Eskola, H. Paukkunen and C. A. Salgado, JHEP 0904 (2009) 065.

[13] R. Sharma, I. Vitev and B. W. Zhang, Phys. Rev. C80 (2009) 054902. Y. He, I. Vitev and B. W. Zhang, arXiv : 1105.2566 [hep-ph] (2011).

[14] W. A. Horowitz and M. Gyulassy, J. Phys. G38 (2011) 124114.

[15] N. Armesto, A. Dainese, C. A. Salgado and U. A. Wiedemann, Phys. Rev. D71 (2005) 054027.

[16] B. Abelev et al. [ALICE Collaboration], arXiv :1305.2707 [nucl-ex] 

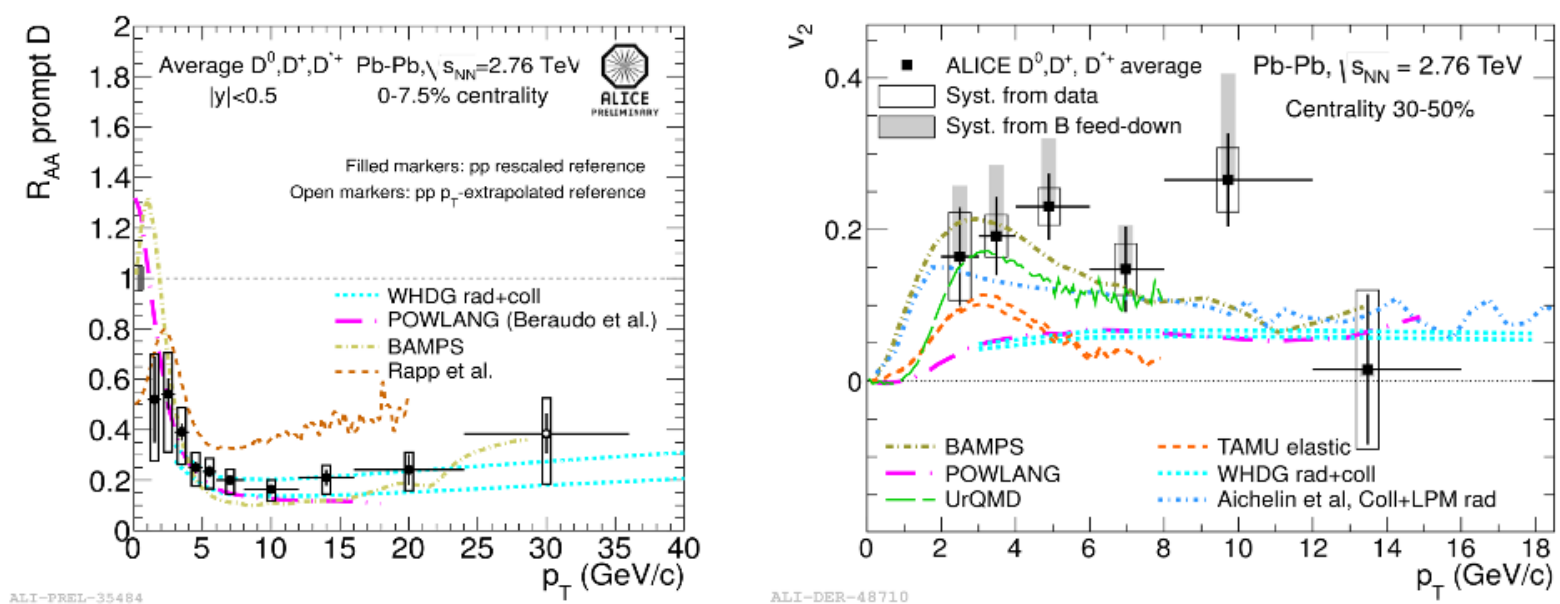

FIGURE 1. D meson $R_{\mathrm{AA}}$ (left) and $v_{2}$ (right) compared with model predictions.
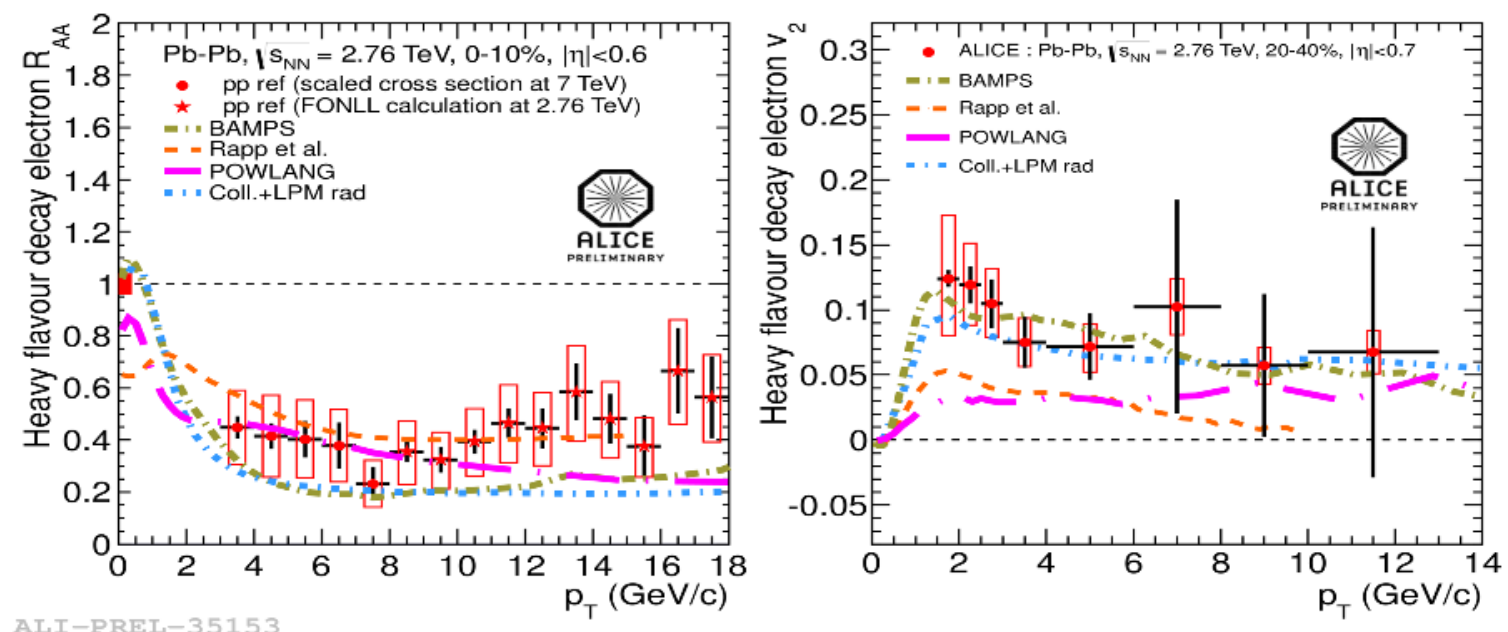

Figure 2. Heavy-flavour decay electron $R_{\mathrm{AA}}$ (left) and $v_{2}$ (right) compared with model predictions.
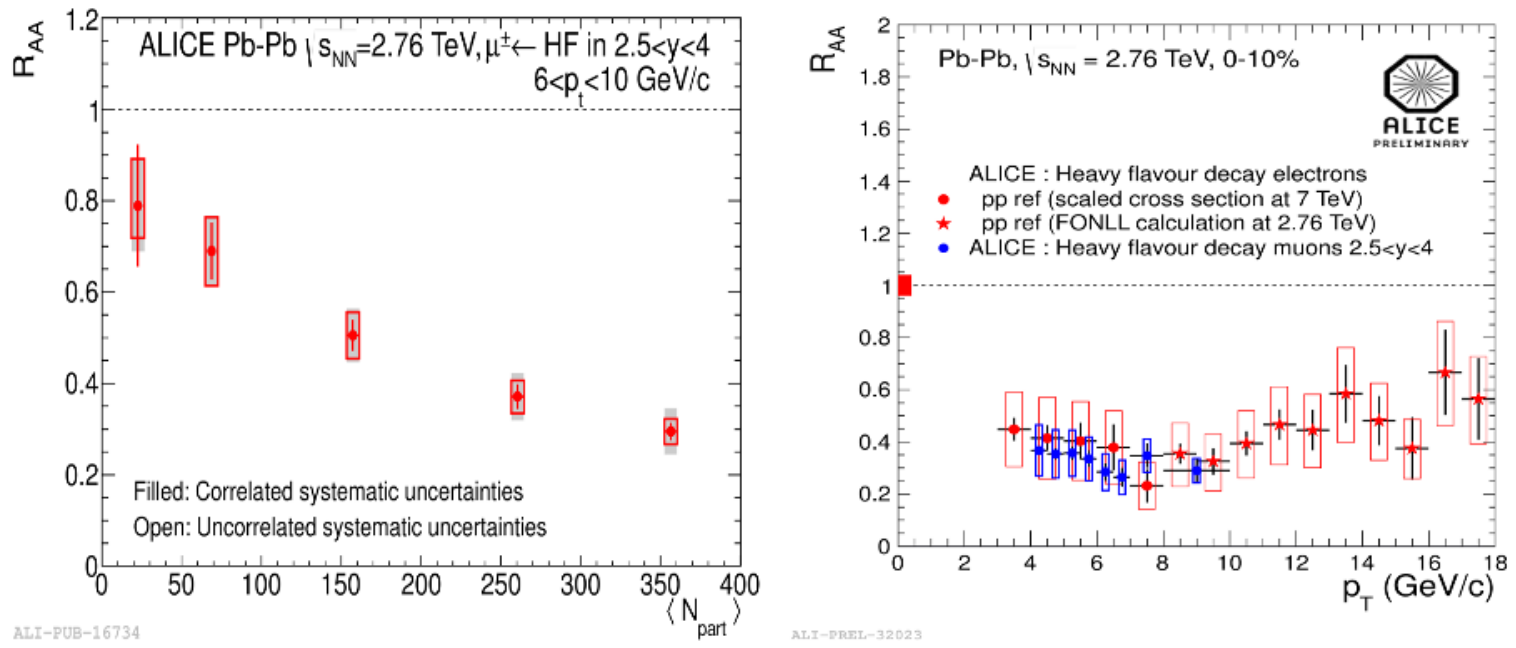

Figure 3. Left : $R_{\mathrm{AA}}$ of heavy-flavour decay muons as a function of centrality; Right : $R_{\mathrm{AA}}$ of heavy-flavour decay muons as a function of $p_{\mathrm{T}}$ (measured in $2.5<y<4$ ) compared to HFE $R_{\mathrm{AA}}$ (Fig.2, left) (measured in $|\eta|<0.6$ ). 

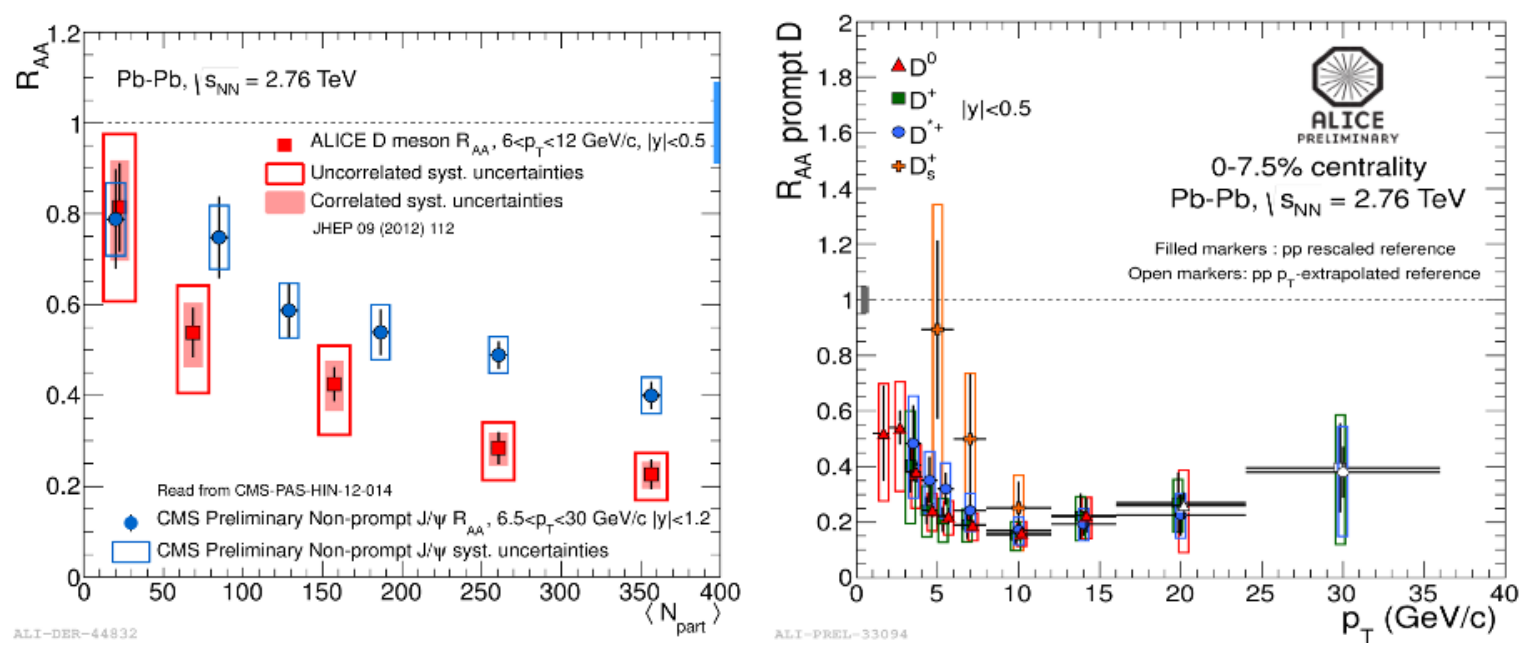

Figure 4. Left : $R_{\mathrm{AA}}$ as a function of centrality of $J / \psi \leftarrow B$ [9] (blue circles) and prompt $\mathrm{D}$ mesons (red squares); Right : $R_{\mathrm{AA}}$ of $\mathrm{D}^{0}$ (red triangle), $\mathrm{D}^{+}$(green square), $\mathrm{D}^{*+}$ (blue circle), and $\mathrm{D}_{\mathrm{s}}^{+}$(orange cross) in $0-7.5 \%$ central $\mathrm{Pb}-\mathrm{Pb}$ collisions. 